\title{
Measuring the Hubble Constant with the Gravitational Lens System CLASS $1608+656$
}

\author{
C. D. Fassnacht, T. J. Pearson, R. D. Blandford, \& A. C. S. Readhead \\ Caltech, Pasadena, CA, U.S.A.
}

Abstract. We present results on the promising new gravitational lens system $1608+656$.

\section{Introduction}

The Cosmic Lens All-Sky Survey (CLASS) is a search for gravitational lenses among flat-spectrum radio sources. Its main goals are to find systems appropriate for measuring $H_{0}$ and to use lensing statistics to put constraints on other cosmological parameters. Details of the survey can be found in Myers et al. (1995) and Browne et al. (1997). Of the five new lenses discovered so far, $1608+656$ is perhaps the most promising for $H_{0}$ measurements.

$1608+656$ consists of four components arranged in a typical lensing geometry. Spectra taken with the Hale $5 \mathrm{~m}$ Telescope gave the redshifts of the lensing galaxy, $z_{\ell}=0.630$ (Myers et al. 1995), and the background source, $z_{s}=1.394$ (Fassnacht et al. 1996). A model of the lensing potential has been constructed using the image positions and flux densities from our VLA observations (Myers et al. 1995). However, since the components are not resolved by the VLA, the lensing potential is only being sampled at four points. Having so few constraints on the model leads to large uncertainties in the predicted time delays and hence in any determination of $H_{0}$ made with this lens.

\section{Constraining the Models}

The lens model can be constrained further by detecting structure in the multiple images of the source. If common components can be identified in each of the images, a transformation matrix from image to image can be constructed, the terms of which are functions of the second derivatives of the lensing potential. We have observed $1608+656$ with the VLBA to search for mas-scale structure in the images. Observations were made at 2,6 , and $18 \mathrm{~cm}$, but no extended structure was seen (e.g., Fig. 1). In spite of the failure to resolve the images, the lens model is constrained more strongly by having more accurate image positions. In addition, HST observations of the system show extended optical emission lensed into arcs (Jackson et al. 1997); these data are being used to refine the lens model.

\section{Monitoring for Time Delays}

To measure a time delay we have monitored $1608+656$ with the VLA between 1996 October 10 and 1997 May 26. Observations were made at $8.4 \mathrm{GHz}$ and were taken, on average, every 4-5 days. The component fluxes are seen to vary at the 3-5\% level. Preliminary time delays have been measured, with component A lagging $\mathrm{B}$ by $\sim 28$ days and $\mathrm{C}$ lagging $\mathrm{B}$ by $\sim 34$ days. The time delays predicted by the current model are $\Delta t_{B A}=14 h^{-1}$ days and $\Delta t_{B C}=19 h^{-1}$ days. Thus, the initial results from this system give $\mathrm{H}_{0} \approx 50-60 \mathrm{~km} \mathrm{sec}^{-1} \mathrm{Mpc}^{-1}$, consistent 


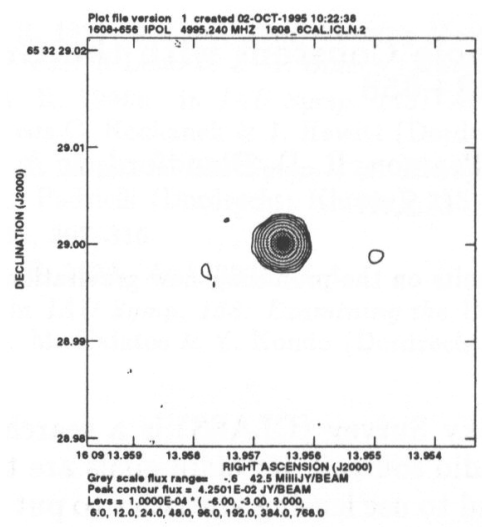

Figure 1. $5 \mathrm{GHz}$ VLBA image of component $\mathrm{A}$ in $1608+656$. The rms noise in the map is $\sim 0.1 \mathrm{mJy}$, giving a dynamic range $>400: 1$. The component is well fitted by a point source; no extended emission is seen. The images of the other components have similar characteristics.

with results found from other lens systems (e.g., Kundić et al. 1997; Schechter et al. 1997; Barkana 1997; Keeton \& Kochanek 1997).

\section{Future Work}

Preliminary results from monitoring $1608+656$ have given a value of $H_{0}$ consistent with that found from other lens systems. However, the uncertainties introduced by errors in the modeling and in the time delay measurements are large $(\sim 20 \%)$. Data from HST observations are being used to put stronger constraints on the lens model, while further monitoring should reduce the uncertainties in the time delays. We are planning on measuring redshifts of other galaxies in the $1608+656$ field to investigate the possibility that a group of galaxies is contributing to the lensing potential. Thus, with further observations this system should yield a solid measurement of $H_{0}$.

Acknowledgments. The National Radio Astronomy Observatory is a facility of the National Science Foundation, operated under a cooperative agreement by Associated Universities, Inc.. Many thanks to the AOC analysts for help with the monitoring observations. This work is supported by the NSF under grant AST 9420018.

\section{References}

Barkana, R. 1997. ApJ, 489, 21-28.

Browne, I. W. A., et al. 1997. To appear in Cosmology from Radio Surveys, eds., M. Bremer \& N. J. F. Jackson.

Fassnacht, C. D., et al. 1996. ApJ, 460, L103-106.

Jackson, N., et al. 1997. $A p J$, submitted.

Keeton, C. R. \& Kochanek, C. S. 1997. ApJ, 47, 42-54.

Kundić, T., et al. 1997. ApJ, 482, 75-82.

Myers, S. T., et al. 1995. $A p J, 447, \mathrm{~L} 5-8$.

Schechter, P., et al. 1997. $A p J, 475$, L85-88. 